\title{
Taxane resistance in prostate cancer is mediated by decreased drug-target engagement
}

\author{
Ada Gjyrezi, ${ }^{1}$ Fang Xie, ${ }^{2}$ Olga Voznesensky, ${ }^{2}$ Prateek Khanna, ${ }^{2}$ Carla Calagua, ${ }^{2}$ Yang Bai, ${ }^{1}$ Justin Kung, ${ }^{3}$ Jim Wu, ${ }^{3}$ Eva Corey, ${ }^{4}$ \\ Bruce Montgomery, ${ }^{5}$ Sandrine Mace, ${ }^{6}$ Diego A. Gianolio, ${ }^{7}$ Glenn J. Bubley, ${ }^{2}$ Steven P. Balk, ${ }^{2}$ Paraskevi Giannakakou, ${ }^{1,8}$ \\ and Rupal S. Bhatt ${ }^{2}$ \\ 'Division of Hematology and Medical Oncology, Department of Medicine, Weill Cornell Medical Center, New York, New York, USA. ²Division of Hematology and Oncology, Department of Medicine, and

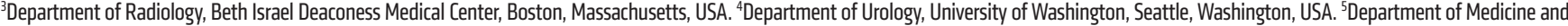 \\ Oncology, University of Washington, Seattle Cancer Care Alliance, Seattle, Washington, USA. ${ }^{6}$ Research and Development, Sanofi, Vitry-sur-Seine, France. ${ }^{7}$ Oncology, Sanofi, Cambridge, Massachusetts, USA. \\ ${ }^{8}$ Sandra and Edward Meyer Cancer Center, Weill Cornell Medical Center, New York, New York, USA.
}

Despite widespread use of taxanes, mechanisms of action and resistance in vivo remain to be established, and there is no way of predicting who will respond to therapy. This study examined prostate cancer (PCa) xenografts and patient samples to identify in vivo mechanisms of taxane action and resistance. Docetaxel drug-target engagement was assessed by confocal anti-tubulin immunofluorescence to quantify microtubule bundling in interphase cells and aberrant mitoses. Tumor biopsies from metastatic PCa patients obtained 2 to 5 days after their first dose of docetaxel or cabazitaxel were processed to assess microtubule bundling, which correlated with clinical response. Microtubule bundling was evident in PCa xenografts 2 to 3 days after docetaxel treatment but was decreased or lost with acquired resistance. Biopsies after treatment with leuprolide plus docetaxel showed extensive microtubule bundling as did biopsies obtained 2 to 3 days after initiation of docetaxel or cabazitaxel in 2 patients with castration-resistant PCa with clinical responses. In contrast, microtubule bundling in biopsies 2 to 3 days after the first dose of docetaxel was markedly lower in 4 nonresponding patients. These findings indicate that taxanes target both mitotic and interphase cells in vivo and that resistance is through mechanisms that impair drug-target engagement. Moreover, the findings suggest that microtubule bundling after initial taxane treatment may be a predictive biomarker for clinical response.

\section{Introduction}

Taxanes are the only line of chemotherapy shown to prolong survival in men with metastatic castration-resistant prostate cancer (CRPC) who have progressed after standard androgen deprivation therapy (ADT, surgical or medical castration) $(1,2)$. Taxanes (docetaxel, cabazitaxel) have not only demonstrated clinical benefits in CRPC, but recent clinical trials have shown unprecedented survival advantages in men with hormone-sensitive metastatic prostate cancer $(\mathrm{PCa})$ who received docetaxel given in combination with standard $\operatorname{ADT}(3,4)$. This survival benefit achieved by the combination of docetaxel and ADT has placed docetaxel in a critical role in castrate-sensitive PCa as well as in CRPC. Despite these clinical benefits of taxane treatment, not all men respond and resistance invariably emerges. Currently, the molecular determinants of clinical response and resistance (intrinsic and acquired) to taxane chemotherapy remain poorly understood.

The mechanism of action of taxanes has been studied extensively in vitro. In cell lines, taxanes bind to tubulin and stabilize microtubules, resulting in extensive reorganization of the fine

Authorship note: AG and FX contributed equally to this work.

Conflict of interest: Sanofi provided funding for this study. DG and SM are Sanofi employees. SPB served as an advisor for Sanofi.

Copyright: (๖) 2020, American Society for Clinical Investigation.

Submitted: July 31, 2019; Accepted: March 11, 2020; Published: May 18, 2020.

Reference information: / Clin Invest. 2020;130(6):3287-3298.

https://doi.org/10.1172/JCl132184. and intricate microtubule network into dense microtubule bundles that can be readily visualized by fluorescence microscopy. Microtubules are highly dynamic filaments fulfilling a number of functions in interphase, such as intracellular trafficking and signaling. They are also critically involved in the formation of the mitotic spindle that facilitates chromosome segregation during mitosis, so that taxane treatment results in aberrant mitotic arrest and apoptotic cell death (5). Although this mitotic arrest is a major mechanism of action in cell culture, there is some controversy as to whether mitotic arrest is the key mechanism underlying the clinical activity of taxanes in patients where the mitotic rate of growing tumors is significantly lower than in cultured cell lines $(6,7)$. Along these lines, we have shown important functions for microtubules in interphase cells, including nuclear translocation of transcription factors $(8,9)$, inhibition of which by taxane treatment can be lethal irrespective of mitosis. Thus, taxane treatment may lead to cell death in mitosis or in interphase (10).

Understanding the mechanism of taxane resistance has been a topic of intense investigation over the last 2 decades. Although there are numerous reports on mechanisms of taxane resistance in preclinical models, there are very few studies addressing the effects of taxanes in vivo in patient tissue $(11,12)$. Possible mechanisms of taxane resistance fall broadly into 2 groups. One group is mechanisms that result in lack of drug-target engagement so that microtubules are not stabilized and microtubule bundles are not formed. One example in this group is alterations in microtu- 

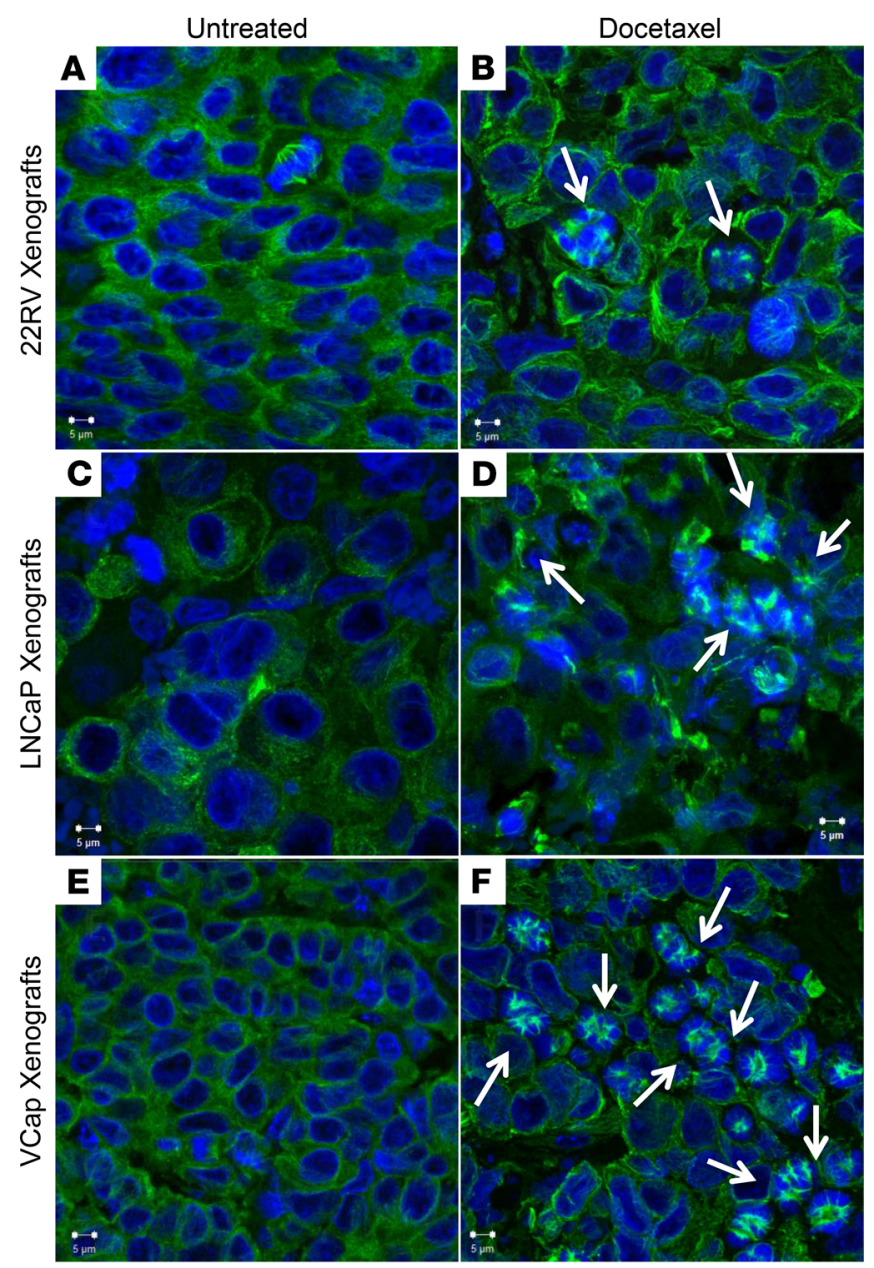

G

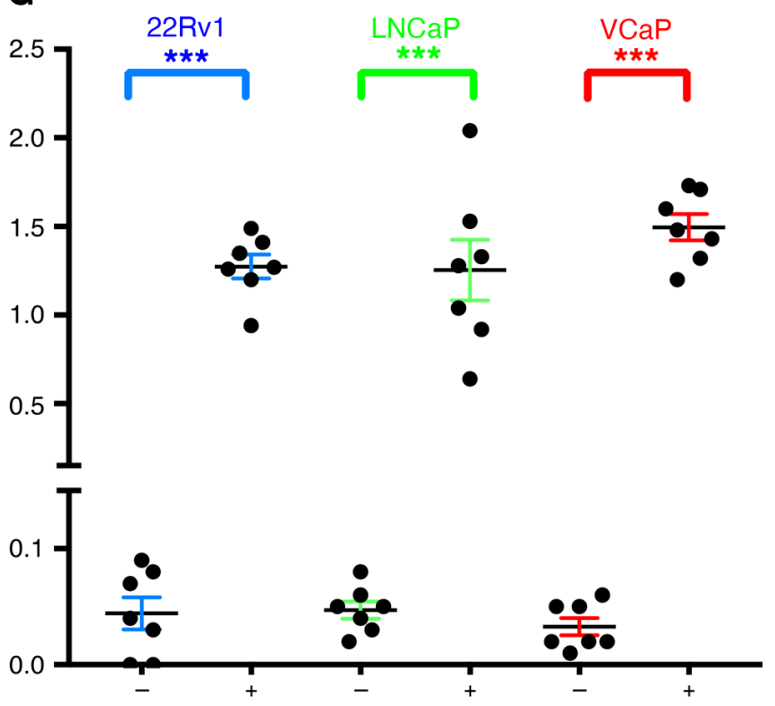

bules that can hinder their taxane-mediated stabilization (13). This includes mutations in beta-tubulin that have been identified in cell lines and have been shown to confer taxane resistance in vitro $(14,15)$, although such mutations have not consistently been noted in patients (16). Tubulin modifications such as acetylation, or altered expression of microtubule-associated proteins, may
Figure 1. Taxane therapy induces microtubule bundling in murine xenograft models. Tubulin bundling as visualized by immunofluorescence (IF) is shown along with quantification via $\mathrm{H}$ score. Three models (CWR22Rv1, LNCaP, and VCaP) are shown. Untreated tumors ( $\mathbf{A}, \mathbf{C}$, and $\mathbf{E})$ show minimal microtubule bundling and tumors from mice treated with docetaxel $(40 \mathrm{mg} /$ kg i.p.) and sacrificed 3 days later (B, D, and $\mathbf{F}$ ) show microtubule bundling in the cytoplasm of interphase cells as well as in mitotic cells. Tubulin is shown in green and DAPI in blue. (C) $\mathrm{H}$ score in treated versus untreated xenografts from each model, graphical representation as dot plot with mean \pm SEM values shown. Mann-Whitney 2-tailed; ${ }^{* *} P<0.001$.

also impair target engagement or prevent stabilization. A second example is increased expression of ATP-binding cassette (ABC) drug efflux transporters that interfere with drug accumulation and thus prevent target engagement (17). In particular, P-glycoprotein expression has been implicated in docetaxel-resistance in PCa (18, 19), whereas the activity of cabazitaxel in patients who have progressed on docetaxel has been partially attributed to the decreased affinity of cabazitaxel for this drug transporter (20). However, trials testing $\mathrm{ABC}$ transporter inhibitors have not demonstrated clinical success to date (21). One further mechanism that may impair target engagement in PCa is ERG overexpression due to the TMPRSS2:ERG gene fusion that occurs in about half of PCa. We have shown that ERG can bind to soluble tubulin and destabilize microtubules by shifting the dynamic equilibrium between soluble and polymerized tubulin toward the soluble pool. As the microtubule polymer is the preferred substrate for taxane binding, ERG overexpression results in relative taxane resistance (22).

The second broad group of resistance mechanisms is alterations in pathways downstream of target engagement that allow cells to tolerate some level of microtubule stabilization. Among this group are increases in BCL2 or MCL1 expression, lack of functional p53, upregulation of Notch, NFkB activation, or increased GATA2-IGF2 (23-26). In addition, we and others have shown that the nuclear transport of the androgen receptor (AR) is dependent on intact, dynamic microtubules whose stabilization by taxanes can inhibit AR nuclear translocation contributing to taxane efficacy in PCa $(27,28)$. This mechanism has been recently validated in a prospective multiinstitutional phase II study of CRPC patients treated with taxanes. Using circulating tumor cells (CTCs) as a source of tumor biopsy, the study showed that decreased nuclear AR in patient CTCs was significantly associated with clinical response to treatment (29). This decrease in nuclear AR is presumably a biomarker indicating effective disruption of microtubule function and downstream pathways. Absence of decreased nuclear AR in patients not responding to taxane treatment may reflect expression of the constitutively active ARv7 splice variant that lacks the microtubule-binding hinge domain and undergoes tubulin-independent transport into the nucleus, which may contribute to taxane resistance in vitro and in vivo $(30,31)$.

Studies of taxanes in vivo are limited, and to our knowledge there are no studies assessing the acute response to taxane treatment in PCa patients. In this study we obtained clinical samples from metastatic PCa patients at approximately 3 days after their initial taxane treatment, and assessed in vivo microtubule bundling as the most direct molecular readout of target engagement in response to taxane therapy. We found that failure to effectively 
A
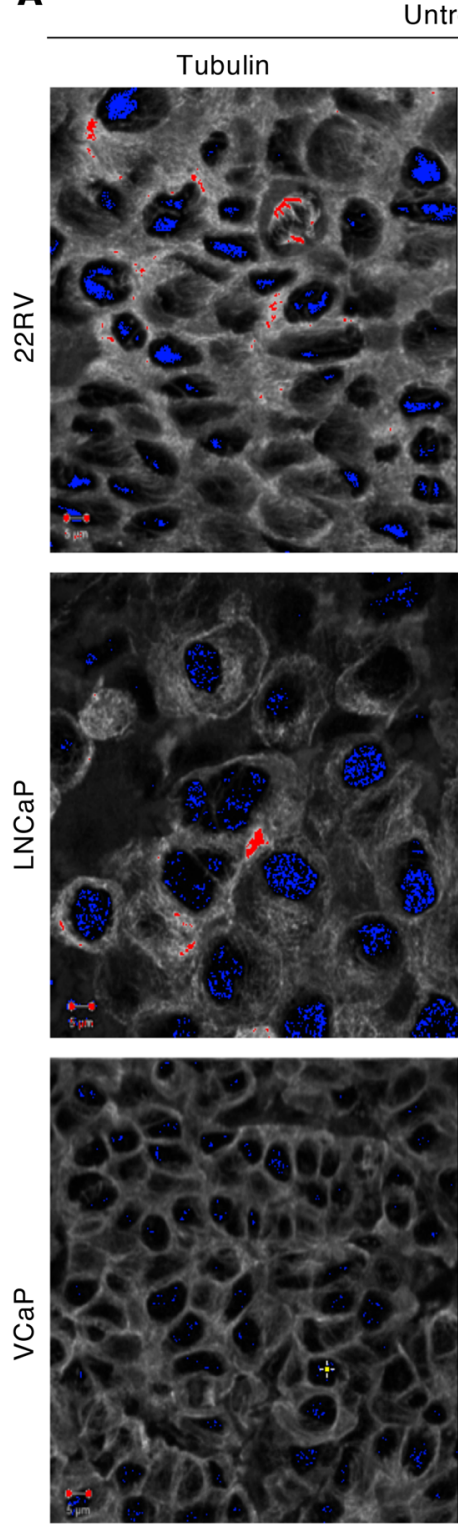

Untreated
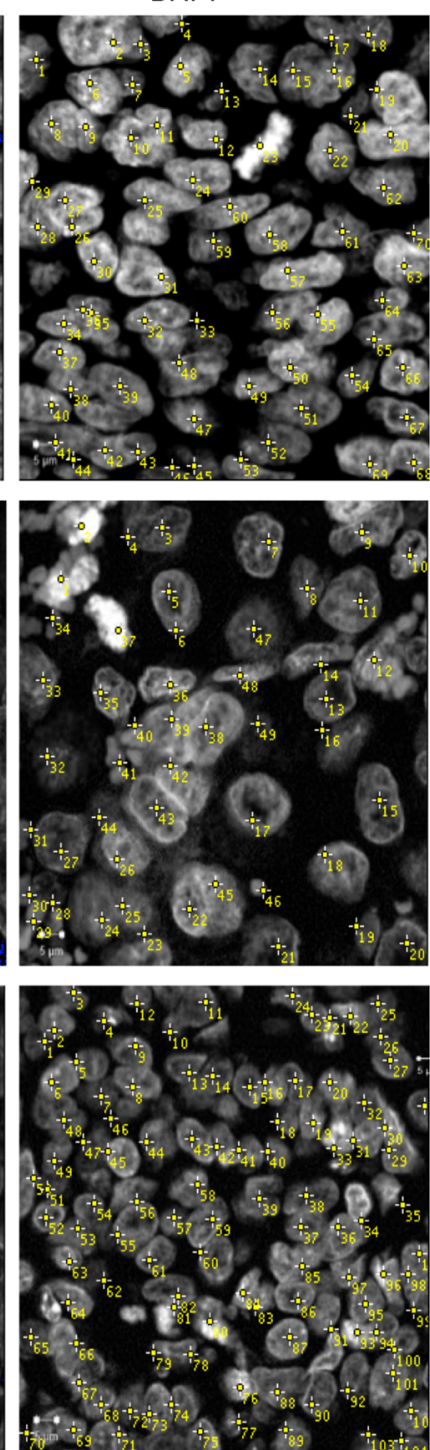

B
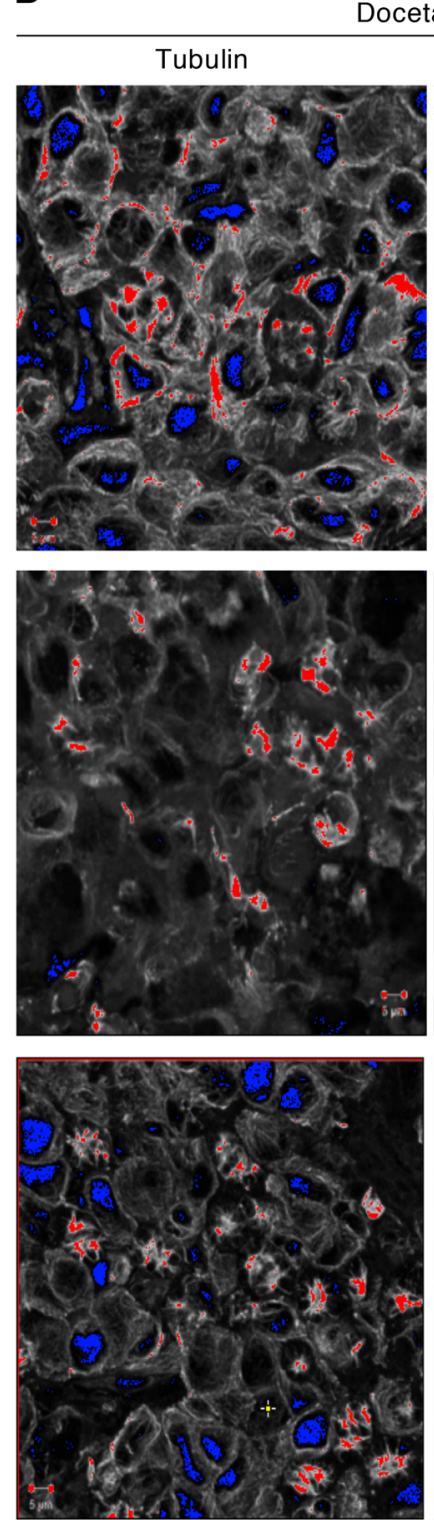

Docetaxel
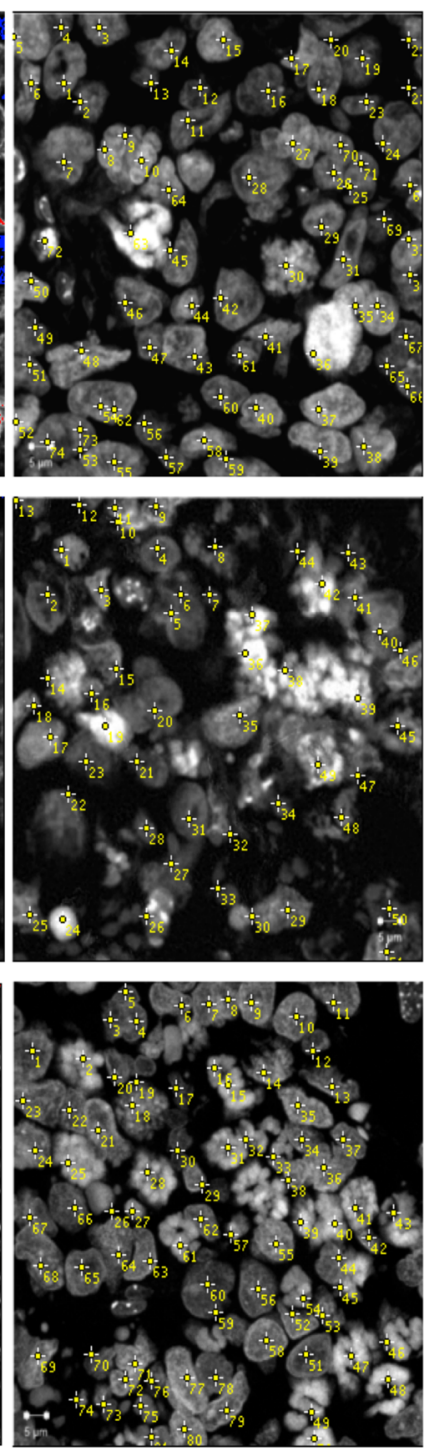

C

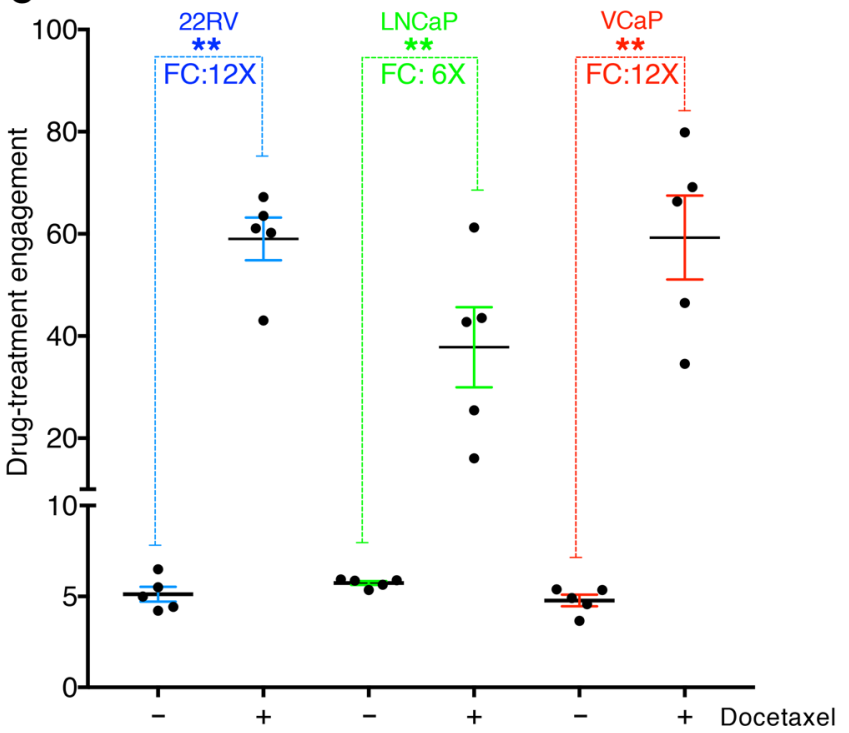

Figure 2. Quantitative assessment of DTE in murine xenograft models. Representative images from untreated tumors $(\mathbf{A})$ and tumors from mice treated with docetaxel (B) were analyzed by a quantitative image analysis platform. Tumors from 3 xenograft models (CWR22Rv1, LNCaP, and $\mathrm{VCaP}$ ) are shown. Tubulin staining is shown in gray scale (white) and the pixels at the top $25 \%$ of integrated fluorescence intensity are marked in red. Individual nuclei stained by DAPI are marked in gray in the DAPI column and marked with yellow numbers. (C) DTE quantitation is displayed as dot plot with mean \pm SEM values. Mann-Whitney 2-tailed; ${ }^{* *} P<0.05$. FC, fold change between treated over control for each xenograft model. The single channel tubulin and DAPI images that correspond to Figure 1, A-F, are shown in $\mathbf{A}$ and $\mathbf{B}$. 
stabilize microtubules was associated with intrinsic taxane resistance in patients with metastatic CRPC and was also associated with acquired resistance in PCa xenograft models. Moreover, in responding patients we primarily observed microtubule bundling in interphase cells with only rare cells arrested in mitosis, suggesting that mitotic arrest may not be the predominant basis for cell death (6). These findings indicate that lack of drug-target engagement, as evidenced by decreased microtubule bundling, which may represent the convergence of many cellular pathways, is a major contributor to taxane resistance. Moreover, they suggest that microtubule bundling could be a potential early predictive biomarker for response to taxane therapy.

\section{Results}

Docetaxel induces microtubule bundling in vivo in PCa xenografts. To assess the in vivo effect of docetaxel on microtubules in $\mathrm{PCa}$, mice bearing VCaP-, LNCaP-, and CWR22Rv1-derived PCa xenografts were treated with a single dose of docetaxel (30 mg/kg i.p.) and microtubule bundling was examined after 3 days by tubulin immunofluorescence (IF) coupled with confocal microscopy to assess spatial rearrangements of the microtubule network. The tubulin staining pattern ranged from fine intricate network microtubule staining that is typical for untreated cells (Figure 1, A, C, and E), to thick cytoplasmic microtubule bundles seen in the treated tumors (Figure 1, B, D, and F). Microtubule bundling was quantified using an algorithm incorporating both cells in mitosis and cells in which bundling occurs in the cytoplasm during interphase (H score) (Figure $1 G$ ). Tumors from untreated mice showed a network of fine and intricate microtubules with $\mathrm{H}$ scores of less than 0.05 (on scale from 0 to 2), and treatment yielded $\mathrm{H}$ scores of 1.25 to 1.43. As expected, a subset of treated cells was arrested in mitosis, with mitotic arrest being prominent in the docetaxel-treated, VCaPderived tumors. LNCaP and CWR22Rv1 had more cytoplasmic microtubule bundling in interphase cells, with CWR22Rv1 showing the most prominent cytoplasmic bundling. The increased mitotic arrest in VCaP did not appear to be due to a higher proliferative rate, as the proliferative rate was similar in CWR22Rv1 and VCaP models (76\% vs. $75 \%$ ) based on Ki67 staining (Supplemental Figure 1; supplemental material available online with this article; https://doi.org/10.1172/JCI132184DS1).

Taxane activity on microtubules is usually qualitatively measured by the extent of microtubule bundling. To obtain a more quantitative measure of effective drug-target engagement (DTE) as a marker of taxane response and resistance, we developed a novel quantification method that avoids any user bias regarding the scoring of microtubule bundles. This image analysis platform calculates the top quartile of fluorescence pixel intensity in the tubulin channel and normalizes for the total number of cells. Figure 2 shows representative images from the PCa xenograft experiment, wherein the tubulin channel, pixels in the top quartile of tubulin fluorescence intensity are marked in red while corresponding individual nuclei are marked in gray with yellow numbers. In untreated cells, the pixels that are in the top 25\% quartile are rare and primarily seen in normally dividing cells or in cell-cell junctions due to excessive shared microtubule density that cannot be assigned to an individual cell (Figure 2A). In contrast, there was a marked increase in the number of pixels marked in red in docetaxel-treated tumors (Figure 2B). The top quartile of pixels in the treated xenografts is indicative of aberrant mitotic arrest and/ or microtubule bundling. Similar to the more qualitative analysis in Figure 1 (H score), DTE in the VCaP tumors was primarily due to aberrant mitoses, whereas interphase bundling was the most prominent DTE feature in the CWR22Rv1 tumors. The accompanying bar graph (Figure 2C) shows a 6- to 12-fold increase in DTE, providing higher accuracy and resolution as compared with the $\mathrm{H}$ score in Figure 1.

Loss of DTE in docetaxel-resistant PCa xenografts. To decipher in vivo mechanisms of taxane resistance, we generated docetaxel-resistant CWR22Rv1 xenografts. In a pilot study, mice bearing CWR22Rv1 xenografts were treated with docetaxel (30 mg/kg i.p., 3 weeks) and assessed for growth. Xenografts showed tumor growth delay compared with untreated tumors after 1 dose. After the second dose, very little tumor growth delay was noted, and tumors continued to grow despite a third dose of docetaxel. Therefore, to elucidate the resistance mechanism, we examined the ability of taxanes to engage their target and induce microtubule bundling at 3 days after the third treatment with docetaxel. Microtubule bundling was compared between these resistant tumors versus sensitive tumors (not previously treated with docetaxel) that were similarly harvested 3 days after docetaxel treatment.

The vehicle-treated sensitive and resistant tumors had occasional cells undergoing normal division (striped arrows, mitotic index $~ 1 \%$ ) and no microtubule bundling (Figure 3, A and B). As expected, there was ample evidence of active drug-target engagement in the sensitive group of mice at 3 days after a single dose of docetaxel. Tumors from these treated sensitive mice had prominent microtubule bundling in interphase cells and to a lesser extent aberrant mitotic cells (Figure 3, D-G, solid arrows). In contrast, docetaxel treatment of the resistant xenografts did not result in any target engagement, as evidenced by the presence of uninterrupted microtubule networks (Figure 3, H-K). Quantitation of these results, by calculating the DTE score, is shown in the corresponding bar graph (Figure 3C). Treatment with docetaxel in the responsive group caused high levels of target engagement. In contrast, tumors from the resistant group had minimal scores similar to the untreated conditions. This result indicates that the basis for resistance in this model is loss of target engagement and suggests that microtubule bundling may represent a novel in vivo predictive biomarker for taxane sensitivity.

To further validate these findings, a second resistance model was developed. The patient-derived castration-resistant LuCaP35CR xenograft line was used to generate tumors in castrated male mice. Mice were then treated with docetaxel or vehicle every 3 weeks. Although the magnitude and duration of response were variable, all treated mice responded and most then progressed after 4 to 6 cycles (Supplemental Figure 2). The mice bearing sensitive (previously vehicle-treated) and resistant tumors were then treated with docetaxel and sacrificed after 3 days to quantitate DTE. Tubulin IF showed minimal microtubule bundling in control tumors (Figure 4, A, D, and G) from mice that did not receive docetaxel, and markedly increased microtubule bundling and aberrant mitoses in the docetaxel-treated sensitive mice (Figure $4, \mathrm{C}, \mathrm{F}$, and I). In contrast, relative to the sensitive tumors, micro- 


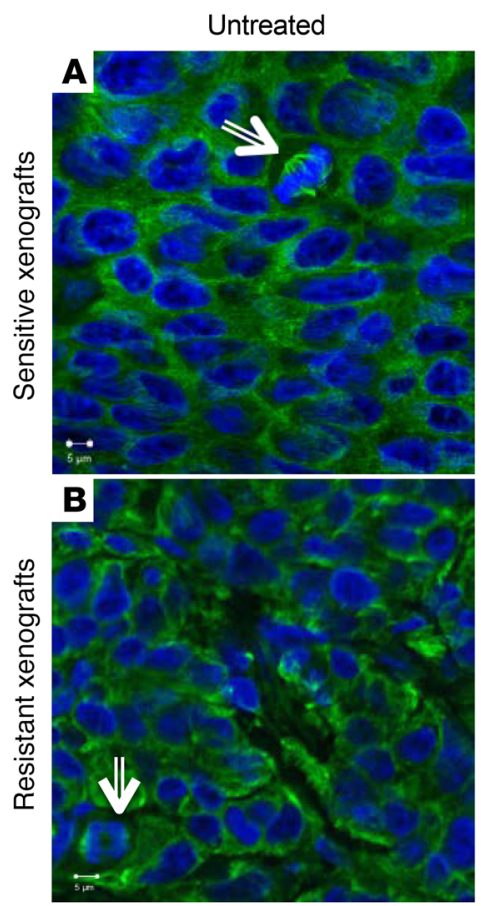

C
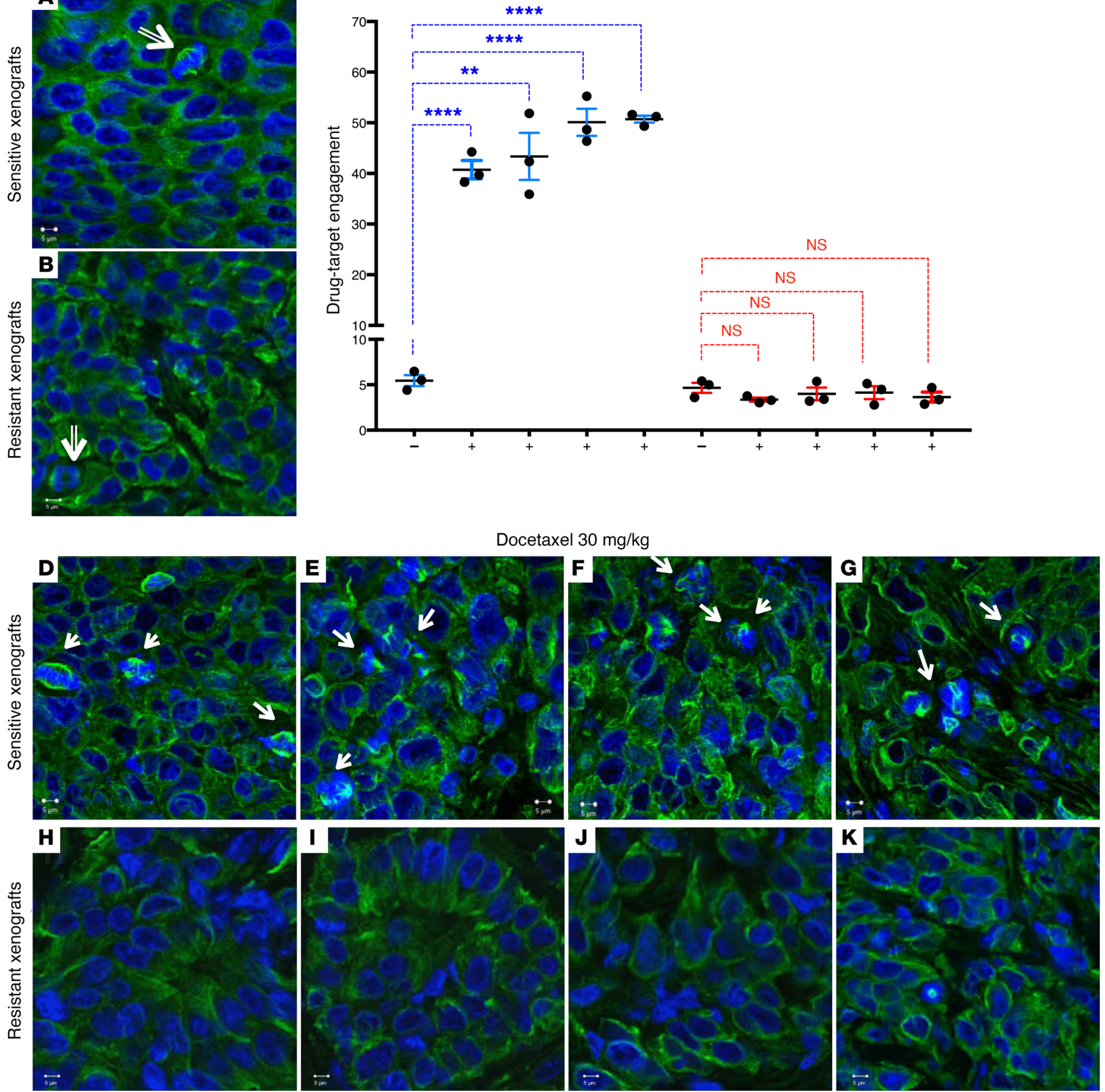

Figure 3. Microtubule bundling is lost in docetaxel-resistant tumors. Mice with CWR22Rv1 docetaxel-sensitive xenografts (A and D-C) were untreated (A) or treated with one dose of docetaxel (30 mg/kg i.p.) and sacrificed after 3 days (D-G). Mice with docetaxel-resistant xenografts were untreated (B) or treated with docetaxel (30 mg/kg i.p.) and sacrificed after 3 days (H-K). (C). Microtubule bundling was assessed by IF and quantified using DTE score and is shown in the accompanying bar graph. DTE quantitation is displayed as dot plot with mean \pm SEM values. Unpaired 2 -tailed $t$ test; ${ }^{* * *} P<0.0001 ;{ }^{* *} P$ $<0.05$. Figure $1 A$ is also shown in $\mathbf{A}$.

tubule bundling was markedly decreased in 2 of 3 resistant tumors (Figure 4, B, E, and H). The results are quantified in Figure 4J and further support the conclusion that loss of target engagement is a prominent basis for acquired docetaxel resistance, although microtubule bundling in 1 resistant tumor clearly shows that additional mechanisms can contribute to resistance.
Microtubule bundling correlates with clinical response to taxanes in patients with metastatic CRPC. To determine the effects of taxane on tubulin in PCa patients, and assess microtubule bundling as a potential biomarker for taxane activity, we enrolled subjects who were initiating taxane therapy on a biopsy tissue acquisition protocol. To assess basal bundling, tubulin staining 

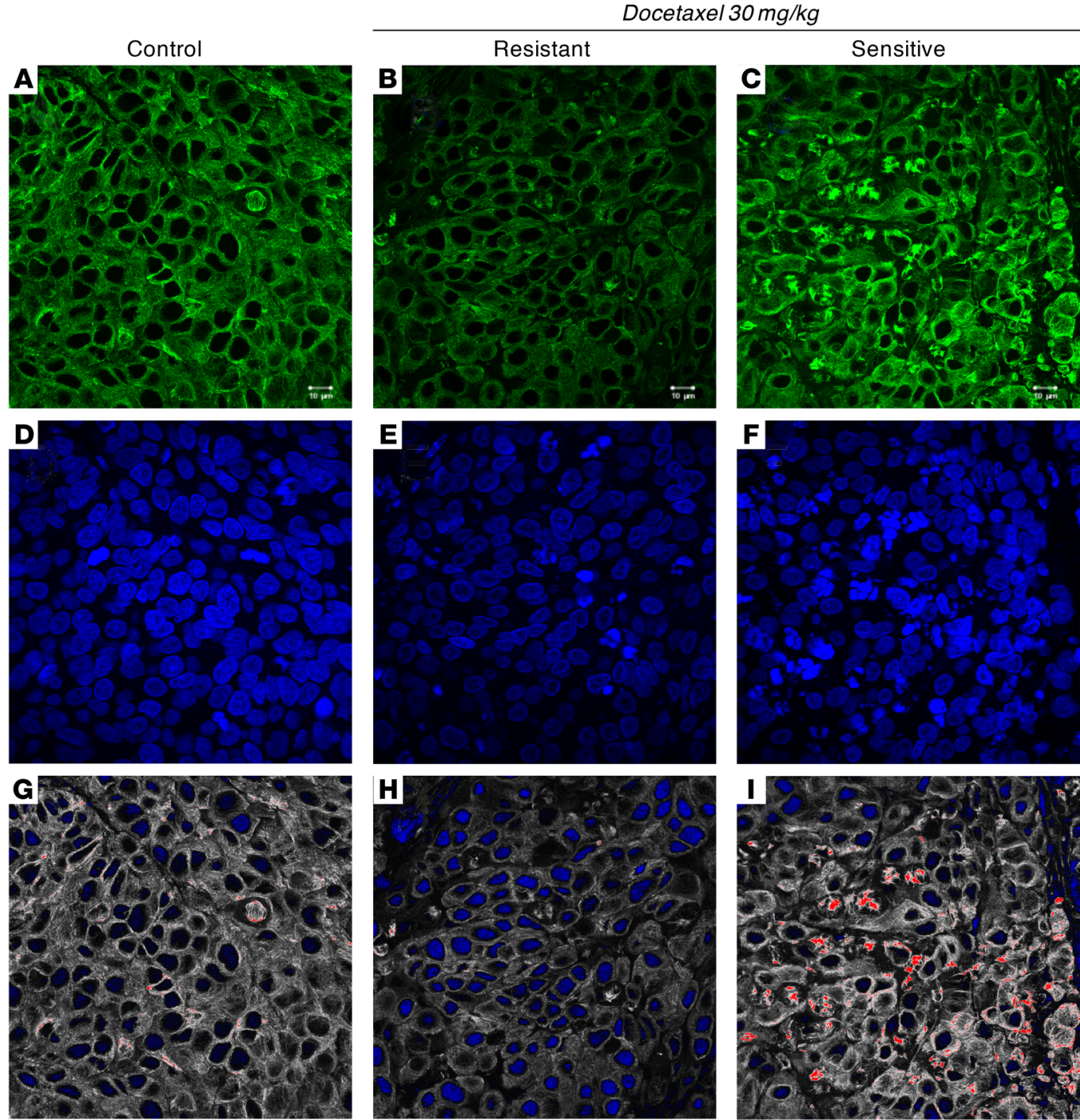

$J$

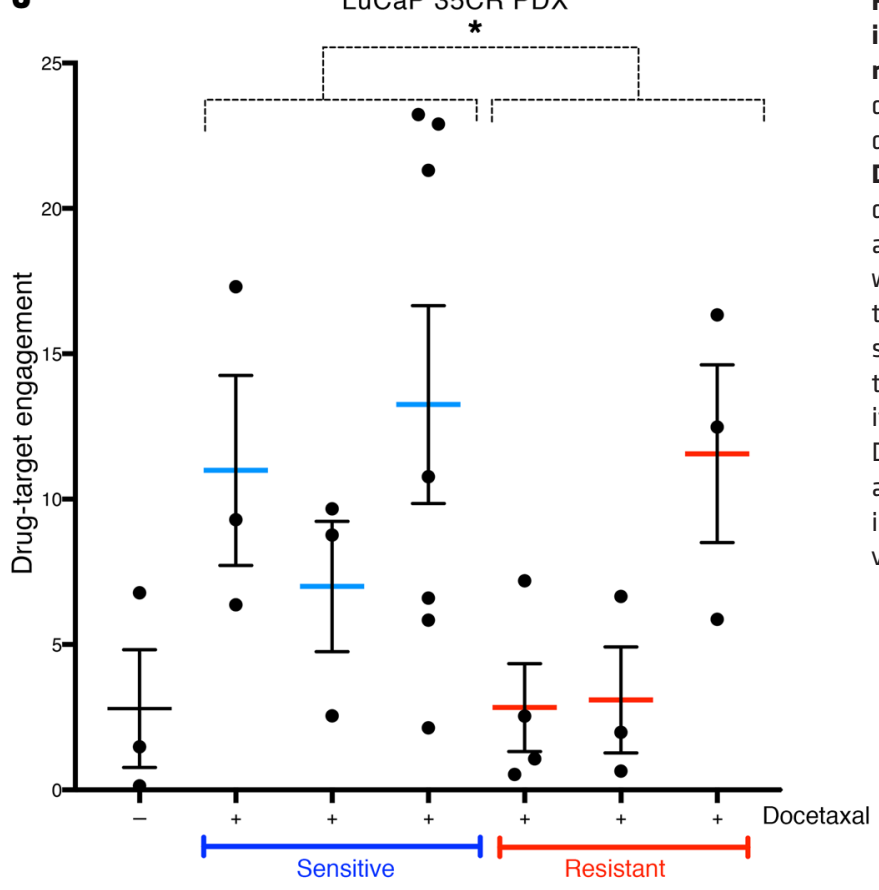

Figure 4. Microtubule bundling is lost in a second patient-derived xenograft model of docetaxel resistance. Mice with docetaxel-sensitive $35 \mathrm{LuCaP}$ patientderived xenografts were untreated (A, D, and $\mathbf{~ C ) ~ o r ~ t r e a t e d ~ w i t h ~ o n e ~ d o s e ~ o f ~}$ docetaxel (30 mg/kg i.p.) and sacrificed after 3 days (B, C, E, F, H, and I). Mice with docetaxel-resistant xenografts were treated with docetaxel $(30 \mathrm{mg} / \mathrm{kg}$ i.p.) and sacrificed after 3 days (B, E, and $\mathbf{H}$ ) and tubulin (A-C), DAPI (D-F), or the composite quantification fields $(\mathbf{G}-\mathbf{I})$ are shown. DTE was quantified and is shown in the accompanying bar graph. DTE quantitation is displayed as dot plot with mean \pm SEM values. Mann-Whitney 2 -tailed; ${ }^{*} P<0.05$. 

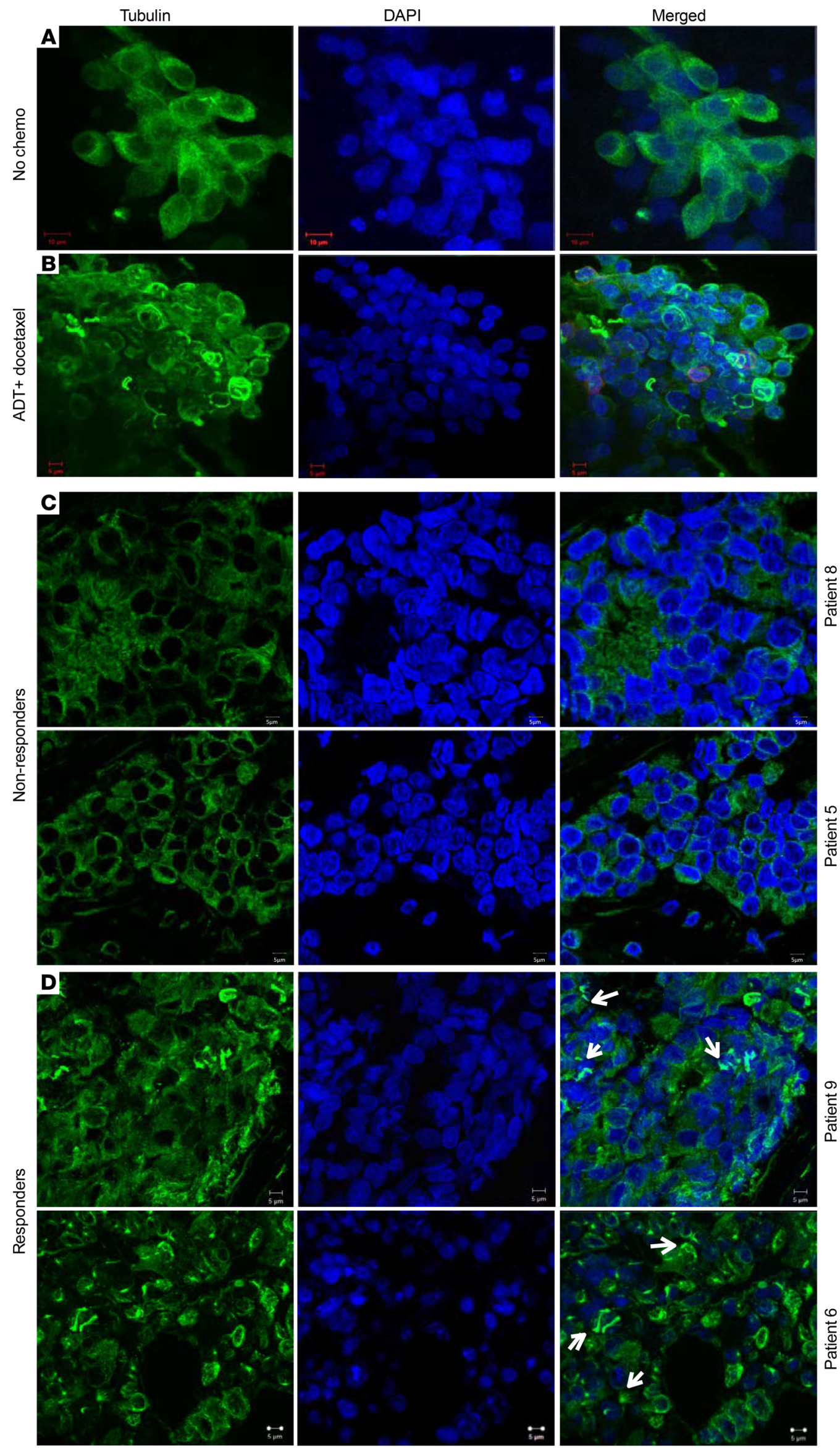

Figure 5. Microtubule bundling can be detected in metastatic PCa patient tumor biopsies and is greater in patients who respond to taxane than in nonresponders. (A) Tubulin IF on a bone marrow tumor biopsy from a CRPC patient who was not treated with chemotherapy. (B) Tubulin IF on a bone marrow metastasis biopsy from a patient with hormone-sensitive metastatic PCa who was treated 5 days earlier with their first dose of leuprolide plus docetaxel.

Bone marrow metastatic tumor biopsies from CRPC patients were obtained at 2 to 3 days after an initial dose of docetaxel, and were analyzed for microtubule bundling by anti-tubulin IF. (C) Representative images from 2 nonresponders. (D) Representative images from the 2 patients with clinical responses (defined by treatment for greater than 6 cycles) 
Table 1. Clinical characteristics of patients with metastatic PCA undergoing biopsy

\begin{tabular}{|c|c|c|c|c|c|c|}
\hline Patient & Prior Rx & Treatment & $\begin{array}{l}\text { Number of days } \\
\text { after chemo }\end{array}$ & Cycles & Overall survival, months & DTE score \\
\hline 1 & Nontaxane clinical trial & None & None & None & $N / A$ & 5.5 \\
\hline 2 & None & Docetaxel + leuprolide & 5 & 6 & Alive & 17.1 \\
\hline 3 & None & Docetaxel + leuprolide & 5 & 6 & Alive & 21.0 \\
\hline 4 & Lup, bical & Docetaxel & 2 & 4 & 9 & 12.0 \\
\hline 5 & $\begin{array}{l}\text { Talc, Pvax, abi, enz, } \\
\text { radium }\end{array}$ & Docetaxel & 2 & 4 & 3 & 9.0 \\
\hline 6 & $\begin{array}{c}\text { Bical, lup, BKM, abi, enz, } \\
\text { radium }\end{array}$ & Docetaxel & 3 & $16^{A}$ & 34 & 16.7 \\
\hline 7 & Lup, provenge, enz & Docetaxel & 3 & 2 & 2 & 9.7 \\
\hline 8 & Lup, abi, rad & Docetaxel & 3 & 3 & 5 & 7.9 \\
\hline 9 & $\begin{array}{l}\text { Lup, keto, tax, abi/ } \\
\text { avodart, enz }\end{array}$ & Cabazitaxel & 2 & 13 & 28 & 15.8 \\
\hline
\end{tabular}

${ }^{A}$ After receiving 16 cycles of docetaxel, patient 6 was also able to get 14 subsequent cycles of cabazitaxel. N/A, not available because patient was on a nontaxane clinical trial for prostate cancer; lup, leuprolide; bical, bicalutamide; Talc, chemohormonal clinical trial; Pvax, prostavax vaccine trial; abi, abiraterone; enz, enzalutamide; BKM, PI3K inhibitor trial; rad, radium-223; keto, ketoconazole; tax, docetaxel; abi/avodart, hormonal clinical trial.

was assessed by IF in a bone metastasis of 1 patient who did not receive any chemotherapy. As expected, tumor from this patient showed a fine and intricate pattern of tubulin staining and had a low DTE score (5.5) (Figure 5A and Table 1). Microtubule bundling was also examined in 2 patients with castration-sensitive metastatic PCa who were initiating therapy with combination docetaxel and leuprolide. Previous studies have shown that taxane clinical responses are greater in hormone-naive PCa and decreased in CRPC and in abiraterone or enzalutamide-resistant PCa. Therefore, it was anticipated that these patients would be more sensitive to taxane therapy. Indeed, bone biopsies obtained from each of these patients at 5 days after their initial dose of docetaxel showed substantial microtubule bundling, with DTE scores of 17.1 and 21.0 (Figure 5B and Table 1).

To assess for correlations between microtubule bundling and clinical responses we enrolled 6 additional patients with metastatic CRPC who were initiating taxane therapy. These patients underwent CT image-guided tumor bone biopsies at 2 to 3 days after their first dose of taxane to evaluate early target engagement by taxane chemotherapy. This timing was based in part on one available previous example in a breast cancer neoadjuvant study that showed apoptotic responses to paclitaxel peaked at 2 to 3 days after treatment (11). Figure 5 shows representative images from patients whose therapy was discontinued after 2 to 4 cycles of taxane treatment (patients 4, 5, 7, and 8) due to lack of response based on imaging and rising serum prostate-specific antigen (Figure 5C) Three of these patients (patients 5, 7, and 8) demonstrated fine cytoplasmic microtubule networks and little microtubule bundling, and had low DTE scores ranging from 7.9 to 9.7 (Table 1). More microtubule bundling was observed in the fourth nonresponder (patient 4), with a DTE score of 12.0 (Table 1). Interestingly, although patient 4 received only 4 cycles of docetaxel and was classified as a nonresponder, his overall survival was 9 months versus 2 to 5 months in the other nonresponders.

In contrast to these 4 patients, the anti-tubulin IF in the 2 responding patients (patients 6 and 9) showed clear evidence of microtubule bundling, which resembled that seen in the docetaxel-sensitive xenografts (Figure 5D). Patient 6 had a DTE score of 16.7 and went on to receive 16 cycles of docetaxel, whereas patient 9 had a DTE score of 15.8 and went on to get 13 cycles of cabazitaxel (Table 1). Overall survival was also greatest in these 2 patients, consistent with previous data showing that the number of cycles of taxane is a predictor of outcome. Of note, patient 9 previously had a prolonged response to docetaxel (16 cycles) before initiating cabazitaxel, whereas patient 6 was able to receive 14 cycles of cabazitaxel after progressing on docetaxel. Despite the small sample size these data suggest that the extent of microtubule bundling after the initial dose of taxane may be an early predictive biomarker for response, and that there is a subset of patients who may be hypersensitive to the effects of microtubule bundling.

The presence of tumor in each biopsy was confirmed by H\&E staining (Supplemental Figure 3) and AR staining (Supplemental Figure 4). Staining for tubulin, AR, DAPI, and merged images are shown for a taxane-sensitive and -resistant patient in Supplemental Figure 4. Although previous studies have shown that the nuclear translocation of AR is dependent on microtubules, and that taxane treatment can decrease AR nuclear accumulation compared with the respective baseline untreated samples $(27,28)$, we observed prominent nuclear AR in both the nonresponders and responders. Similar to our findings with patient samples, staining for AR and tubulin in docetaxel-treated murine xenografts also showed prominent nuclear as well as some cytoplasmic AR (Supplemental Figure 5). Of note, previous studies have found that nuclear transport of the ARv7 splice variant is not dependent on microtubules $(30,31)$, suggesting that the nuclear AR in taxane-treated tumors may be ARv7 rather than the full-length AR. However, using an AR C-terminal-specific antibody to detect the full-length AR, we found that the full-length AR remained primarily nuclear after docetaxel treatment in 3 docetaxel-responsive PCa xenograft models (Supplemental Figure 6). Not surprisingly, full-length AR was also predominantly nuclear after docetaxel treatment in the corresponding xenografts with acquired docetaxel resistance 
(Supplemental Figure 6). ARv7 expression was also nuclear in the untreated and treated tumors, although in comparison to fulllength AR that was highly expressed in all tumors cells, ARv7 was detected in only a minor fraction of cells (Supplemental Figure 6).

Consistent with these patient-derived xenograft (PDX) results, we found predominantly intense nuclear expression of the fulllength AR in each patient tumor biopsy we were able to examine, including in 1 tumor with very high levels of microtubule bundling (DTE score 21.0) (Supplemental Figure 7). ARv7 expression was found in 2 of the tumor biopsies and, as expected, was predominantly nuclear. Taken together, these findings demonstrate that the nuclear AR expression in tumor tissue after taxane treatment does not solely reflect ARv7, and that the therapy is not preventing the nuclear translocation of full-length AR in tumor tissue. However, in the absence of matching baseline tumor biopsies, changes in nuclear AR following taxane treatment cannot be quantified.

These data show the tight correlation between tumor DTE and response to taxane treatment in xenografts and PCa patients and suggest that early evaluation of microtubule bundling could be predictive of taxane activity and could be used to customize treatment for the individual patient.

\section{Discussion}

Despite their widespread use, the actions of taxanes in vivo have not been fully characterized, the mechanisms that mediate intrinsic or acquired resistance remain to be established, and there is no current way of predicting who will respond to therapy. In this study, we sought to elucidate in vivo taxane mechanisms of action and resistance in PCa. We showed in mouse xenograft models and in tumor biopsies from patients that taxane target engagement assessed by microtubule bundling in interphase cells and aberrant mitotic arrest was associated with response to therapy. In 4 mouse PCa xenograft models, docetaxel induced microtubule bundling acutely after an initial treatment. In contrast, this microtubule bundling response was lost in CWR22Rv1 xenografts that showed resistance after 3 cycles of docetaxel, and was similarly reduced in the LuCaP35CR xenograft model. Microtubule bundling, which was predominantly in interphase cells, was also observed in tumor biopsies taken from patients acutely after their initial taxane treatment. Importantly, the extent of microtubule bundling was associated with clinical response in these patients. Together, these findings indicate that taxanes target primarily interphase cells in vivo, and that resistance is due to mechanisms that impair target engagement, as assessed by loss of microtubule bundling. Moreover, the findings suggest that microtubule bundling after an initial taxane treatment may be an early predictive biomarker for clinical response.

In vitro studies clearly show that taxane-mediated microtubule stabilization causes inefficient spindle separation, and that prolonged or arrested mitosis then leads to apoptotic cell death. However, although the mitotic spindle appears to be most sensitive to taxanes in actively dividing cells, these drugs can also stabilize microtubules in interphase cells (where cells spend the majority of the cell cycle), and thereby impair intracellular trafficking of organelles, vesicles, and proteins $(8,9)$. Previous studies analyzing cell fate in response to taxanes in single cells from different cell lines have shown that cells can die from either mitotic arrest or in interphase based on distinct pathways (10). In the mouse xenograft models we found a spectrum of responses from predominant mitotic arrest in the VCaP model to more pronounced interphase cytoplasmic microtubule bundling in the LNCaP and CWR22Rv1 models. The basis for this difference is not clear, and does not appear to be due to differences in proliferation rate. Interestingly, we have previously reported that ERG, which is highly expressed in the VCaP model due to TMPRSS2:ERG fusion, binds tubulin and decreases sensitivity to taxanes (22). This could possibly result in decreased effects on microtubules in interphase cells versus mitotic cells.

The clinical success of drugs that interfere with normal microtubule function, either by stabilization (taxanes) or depolymerization (vinca alkaloids, eribulin), has been attributed primarily to their antimitotic effects. However, the lack of clinical activity of antimitotic agents that do not affect microtubules, such as the mitotic-kinase inhibitors, together with our increasing understanding of the crucial role of the microtubule network in interphase, has challenged the antimitotic-centric view regarding the mechanism of action of taxanes in patients $(6,7)$. Consistent with this notion, our data using patient biopsies have primarily revealed microtubule bundling in interphase cells. However, it should be emphasized that we examined only a single time point (2-3 days after treatment), and cannot rule out an earlier wave of mitotic arrest and apoptosis that may occur within 24 hours after therapy $(11,12)$. Nonetheless, these findings suggest that microtubule stabilization in interphase cells is making a substantial or major contribution to efficacy.

Taxane resistance (intrinsic or acquired) may broadly reflect lack of target engagement due to mechanisms upstream of target engagement, such as increases in drug-efflux transporters, or mechanisms that impair the ability of taxane to stabilize microtubules $(13,16-18,32-34)$. In addition to tubulin mutations (which appear to be relatively uncommon in clinical samples), these latter mechanisms may include altered phosphorylation of tubulins or of microtubule-associated proteins. Indeed, the tyrosine kinase SYK has been reported to be increased in ovarian cancers that relapse after paclitaxel and to phosphorylate $\alpha$ - and $\beta$-tubulins and several microtubule-associated proteins, with SYK inhibition combined with paclitaxel being found to stabilize microtubules in paclitaxel-resistant cells (35). In another study, the tyrosine kinase FER was found to phosphorylate the microtubule-associated protein CRMP2 and thereby decrease its ability to induce microtubule bundling, so that FER inhibition could sensitize ovarian cancer cells to paclitaxel (36). Additional kinases targeting microtubule-associated proteins have also been reported to modulate the effects of taxanes (37).

Alternatively, resistance may reflect alterations in pathways downstream of microtubule bundling that allow the cells to survive some level of microtubule stabilization (23-26, 38, 39). Our results in the CWR22Rv1 and LuCaP35CR models show that loss of target engagement contributes to resistance. Further studies are underway in these and additional in vivo models to elucidate the specific mechanisms of in vivo taxane resistance and translate these findings to the clinic. Notably, our results in PCa patients also support loss of target engagement as a resistance mechanism, as decreased microtubule bundling was associated with clinical 
drug resistance. Nonetheless, it should be emphasized that alterations in downstream survival pathways, which may also contribute to metastatic growth and resistance to AR-targeted therapies, likely contribute to taxane resistance by allowing cells to better tolerate some level of microtubule stabilization.

With respect to taxanes in PCa specifically, we and others have shown that nuclear transport of full-length AR uses functional microtubules and is impaired by taxane-induced microtubule stabilization, likely contributing to taxane efficacy in PCa $(27,28)$. This mechanism was clinically validated in a prospective clinical trial where decreased AR nuclear accumulation in CRPC patient CTCs was correlated with response to treatment (29). Moreover, we and others showed that ARv7, the most prevalent AR splice variant, undergoes tubulin-independent nuclear import, which may in some cases bypass the need for functional microtubules and contribute to taxane resistance $(30,31,40)$. Significantly, we did not observe clear differences in nuclear expression of fulllength AR in docetaxel-treated versus untreated PCa xenografts, and found predominantly nuclear full-length AR in the biopsy samples from docetaxel-treated patients. However, the absence of the matching baseline biopsies from the patients precludes firm conclusions, particularly as the clinical trial showed that the decrease in nuclear AR between baseline and on-treatment samples was correlated with clinical response, as opposed to absolute nuclear AR levels at a single time point (29). In addition, differences in CTCs versus bulk tumor could explain the observed discrepancies due to intratumor heterogeneity or distinct biological properties of CTCs (41).

There are limitations to our study. Our patient sample size was small, as it was challenging to coordinate and obtain biopsies of bone metastases within a short time after initiation of chemotherapy. We also had only 1 time point per patient, so we were not able to assess treatment-induced changes in tumors over time, or even compare directly pre- and posttreatment samples. The use of CTCs to longitudinally monitor patient response by evaluation of DTE could help alleviate this challenge in future clinical studies. Toward that goal, we adapted our DTE assay to CTC microtubules and it shows promising results in identifying microtubule bundling following taxane treatment (Supplemental Figure 8).

Our study provides evidence for DTE being a critical determinant of clinical response to taxane chemotherapy. Interestingly, the most dramatic microtubule bundling was noted in the patient with a marked response to cabazitaxel, a taxane shown to be active in the setting of docetaxel resistance as well as shown to be more active than docetaxel in inducing tubulin polymerization in vitro. Our clinical data, together with the reported feasibility of these studies in circulating tumor cells (29), indicate that future clinical studies should incorporate microtubule bundling as a molecular endpoint of response. In addition, our results indicate that preclinical studies and clinical trials need to focus on mechanisms that may be impairing target engagement, and therapeutic approaches that may enhance taxane-mediated microtubule stabilization to improve clinical outcomes. Finally, while our data identify target engagement as a critical determinant of taxane response, it is clear that additional downstream mechanisms may determine the ability of cells to tolerate some degree of tubulin stabilization and thereby contribute to resistance. Identification and targeting of these downstream mechanisms may also provide novel therapeutic options and improve responses.

\section{Methods}

Murine xenograft generation and treatment. Male nude mice (Taconic Biosciences, Inc., model NCRNU) were injected subcutaneously with approximately 106 cells from VCaP, LNCaP, and CWR22Rv1 human PCa cell lines in 50\% Matrigel. Cells were obtained from ATCC, authenticated by short tandem repeat profiling, and were free of mycoplasma. Tumors were measured 3 times per week, and treatment was initiated when the long axis of the tumors measured 10 $\mathrm{mm}$. Docetaxel was obtained from the Beth Israel Deaconess Medical Center (BIDMC) clinical pharmacy. Mice were treated with the stated doses (30 or $40 \mathrm{mg} / \mathrm{kg}$ i.p.) and tumors were harvested 2 to 3 days after treatment. For the resistant model, mice bearing CWR22Rv1 cell-derived tumors were treated with $30 \mathrm{mg} / \mathrm{kg}$ i.p. docetaxel every 3 weeks, and tumors were harvested 2 days after the third docetaxel dose. Additionally, the castration-resistant LuCaP35CR PDX tumor (obtained from Eva Corey, University of Washington, Seattle, Washington) propagated in castrated male mice was used to generate a second in vivo model of docetaxel resistance (42). Subcutaneous LuCaP35CR tumors were propagated and treated with docetaxel at the same dose and schedule as above for CWR22Rv1 until no response was seen and tumor grew despite 4 to 6 cycles of the therapy. One last dose was then administered and tumors were harvested 2 to 3 days after this last dose. Tumors were fixed in formalin and embedded in paraffin.

Patient selection and biopsy collection. Patients were recruited from the Genitourinary Cancer clinics at BIDMC and the University of Washington. Patients with metastatic PCa who were scheduled to receive docetaxel- or cabazitaxel-based chemotherapy were enrolled on an IRB-approved tissue collection protocol. Consent to obtain a bone biopsy at 2 to 5 days after the first dose of taxane was obtained. Bone metastases were biopsied under image guidance and fixed at room temperature for 1 hour in 2\% formaldehyde in PHEM buffer (PIPES 60 mM, HEPES 25 mM, EGTA 10 mM, $\mathrm{MgCl}_{2} 2 \mathrm{mM}$ ) immediately after they were collected from the patient and then transferred into a vial containing $4 \%$ formaldehyde in $0.1 \mathrm{M}$ sodium cacodylate for 4 hours at room temperature, followed by paraffin embedding. Clinical follow up was performed per routine standard of care, and number of cycles was used as a metric for response to treatment.

Immunofluorescence. Murine tumor and patient biopsy blocks were cut into $5-\mu \mathrm{m}$ thick sections. Slides were baked for 30 minutes at $60^{\circ} \mathrm{C}$ before staining. Slides were deparaffinized and hydrated through xylene and graded alcohol series and washed in PBS 3 times. Antigen retrieval was done for 30 minutes in EnVision FLEX Target retrieval solution High PH (Dako, DM828), followed by 3 washes in $0.3 \%$ Triton $\mathrm{X}-100$ in PBS. Blocking was performed by incubating the slides in $10 \%$ goat serum in PBS for 1 hour at room temperature. Anti- $\alpha$-tubulin rat monoclonal antibody, clone YL1/2 (MilliporeSigma, Mab1864-I) was diluted 1:100 in blocking buffer. Alexa Fluor 488-conjugated secondary goat anti-rat antibody (Thermo Fisher Scientific, A-11006) then was used at 1:500 dilution in blocking buffer. For AR IF, AR N-20 rabbit antibody (Santa Cruz Biotechnology, sc-816X) was used at dilution 1:2500 in blocking buffer. Incubation was carried for 24 hours at $4^{\circ} \mathrm{C}$, followed by 3 washes in PBS. Alexa Fluor 594-conjugated secondary goat anti-rabbit antibody (Thermo Fisher Scientific, A-11012) then was used at dilution 1:500 in blocking buffer. Slides were incubated 
with secondary antibody for 1 hour at room temperature, followed by 3 washes in PBS. Nuclear counterstaining was performed with DAPI incubation for 5 minutes at room temperature followed by 3 PBS washes. For patient biopsy samples, slides were immersed for $30 \mathrm{~min}$ utes into 70\% ethanol, 0.1\% Sudan Black B (Harleco, 3545-12) after fluorescent labeling to minimize auto fluorescence. The excess Sudan Black B solution was gently wiped off and slides were washed in PBS. All stained slides were cover slipped, protected from light, and kept at $-20^{\circ} \mathrm{C}$ for longer storage. Images were acquired with a Zeiss LSM 700 confocal microscope under a $\times 63 / 1.4$ NA objective.

Manual scoring of the mouse xenografts and patient biopsies. A microtubule (MT) H score index was developed to determine and quantify DTE in tumor biopsy samples. The $\mathrm{H}$ index is calculated by multiplying the percentage of cells with active DTE (cells with aberrant mitoses plus cells with microtubule bundling) by the bundling intensity. The $\mathrm{H}$ index is on a scale of 0 to 2 , where 0 means no engagement, 1 moderate engagement, and 2 maximum target engagement.

Image analysis and quantification of the DTE. A second semiautomated scoring algorithm was developed to quantify DTE. This methodology avoids qualitative, operator-based assessment of MT bundling, and uses the fluorescence pixel intensity distribution for each image. Briefly, following image acquisition, using identical confocal microscopy acquisition settings, laser intensities, aperture, etc., we analyzed several representative images for each sample using Image J, which is a Java-based open-source image processing software developed and made available by the NIH. Then we quantified fluorescence intensity for the tubulin channel in the entire image, a method that requires prior background subtraction to correct for potentially uneven illumination, followed by integrated intensity calculation, which takes into account all pixels within the region of interest. The pixel distribution was obtained for each image with a minimum of 0 to a maximum of 255 pixel value. For each image we calculated the number of pixels at the top quartile of fluorescence intensity. For DTE determination we selected the number of pixels at the top $25 \%$ of integrated intensity and normalized by the total cell number, which was quantified by the DAPI nuclear stain.

Immunohistochemistry. For AR and ARv7 IHC, slides were baked for 30 minutes at $60^{\circ} \mathrm{C}$ before staining, deparaffinized, and hydrated through xylene and graded alcohol series and washed in PBS 3 times. Antigen retrieval was done in Diva Decloaker DV2004MX boiling buffer (Biocare Medical, 100810) in a steamer for 30 minutes, then cooled for 20 minutes inside the steamer and for 5 minutes at room temperature. Endogenous peroxidase blocking was done by incubating the slides in 3\% hydrogen peroxide for 5 minutes. IHC was done using VECTASTAIN rabbit or mouse Elite ABC kit (Vector Laboratories, PK-6101 and PK-6102) following the manufacturer's protocol. For total AR, slides were incubated with AR antibody (clone 441) (Santa Cruz Biotechnology, sc-7305) diluted 1:50 for 1 hour at room temperature. For AR C-terminus, slides were incubated with AR Cterminal-specific antibody (Abcam, ab227678 [SP242]) diluted 1:200 for 1 hour at room temperature. For ARv7, slides were incubated with ARv7 specific antibody (RevMab, clone RM7) diluted 1:2000 for 1 hour at room temperature. Chromogen detection was performed using Vector Laboratories peroxidase substrate kit (DAB SK-4100).

Statistics. DTE quantitation is displayed as dot plot with mean \pm SEM values. Mann-Whitney tests were applied to the data and details of each analysis including significance threshold are listed in each figure. Overall significance threshold is $P$ less than 0.05 .

Study approval. Patients were recruited from the Genitourinary Cancer clinics at BIDMC and the University of Washington with approval from corresponding IRBs. All animal experiments were approved by the BIDMC Institutional Animal Care and Use Committee and were performed in accordance with institutional and national guidelines.

\section{Author contributions}

AG performed the immunofluorescence for microtubule bundling and the DTE quantification and worked on the manuscript and figures. FX performed the murine studies of taxane-resistant prostate cancer and worked on the related part of the manuscript and figures. PK assisted with the murine tissue studies and worked on the related part of the manuscript. CC performed the immunohistochemistry for androgen receptor with quantification. YB assisted with DTE quantification. JK and JW performed the image-guided biopsies of metastatic prostate cancer in patients. EC provided the patient-derived xenograft models. BM participated in patient accrual for the biopsy of metastatic lesions. SM and DAG initiated the study, helped develop the experimental plan, and provided funding for the study. GJB participated in patient accrual for the biopsy of metastatic lesions. OV performed murine studies, prepared biopsy samples, and assisted in DTE assessment. RSB, SPB, and PG initiated the study, coordinated experiments, planned the experimental settings, arranged figure settings, and edited the manuscript.

\section{Acknowledgments}

Funding for the study was provided by Sanofi. RSB was also supported by NIH R01 CA196996. SPB was supported by NIH P01 CA163227 and SPORE P50 CA090381 and the Bridge Project (partnership between the Koch Institute for Integrative Cancer Research at MIT and the Dana-Farber Cancer Institute at Harvard Medical School). PG was supported by NCI R01 CA179100, NCI SPORE in Prostate Cancer 1P50 CA211024, NCI R21 CA216800, and Department of Defense PC180637.

Address correspondence to: Rupal S. Bhatt, Division of Hematology and Oncology, Beth Israel Deaconess Medical Center, 330 Brookline Avenue, Boston, Massachusetts 02215, USA. Email: rbhatt@bidmc.harvard.edu. Or to: Paraskevi Giannakakou, Division of Hematology \& Medical Oncology, Department of Medicine, Weill Cornell Medical Center, New York, New York 10065, USA. Email: pag2015@med.cornell.edu. Or to: Steven Balk, Division of Hematology and Oncology, Beth Israel Deaconess Medical Center, 330 Brookline Avenue, Boston, Massachusetts 02215, USA.Email:sbalk@bidmc.harvard.edu.
1. Tannock IF, et al. Docetaxel plus prednisone or mitoxantrone plus prednisone for advanced prostate cancer. N Engl JMed. 2004;351(15):1502-1512.

2. Petrylak DP, et al. Docetaxel and estramustine compared with mitoxantrone and prednisone for advanced refractory prostate cancer. $\mathrm{N} \mathrm{EnglJ}$ Med. 2004;351(15):1513-1520.

3. Sweeney CJ, et al. Chemohormonal therapy in metastatic hormone-sensitive prostate cancer. N Engl JMed. 2015;373(8):737-746.

4. James ND, et al. Addition of docetaxel, zoledronic acid, or both to first-line long-term hormone 
therapy in prostate cancer (STAMPEDE): survival results from an adaptive, multiarm, multistage, platform randomised controlled trial. Lancet. 2016;387(10024):1163-1177.

5. Jordan MA, Wilson L. Microtubules as a target for anticancer drugs. Nat Rev Cancer. 2004;4(4):253-265.

6. Komlodi-Pasztor E, Sackett D, Wilkerson J, Fojo T. Mitosis is not a key target of microtubule agents in patient tumors. Nat Rev Clin Oncol. 2011;8(4):244-250.

7. Shi J, Mitchison TJ. Cell death response to anti-mitotic drug treatment in cell culture, mouse tumor model and the clinic. Endocr Relat Cancer. 2017;24(9):T83-T96.

8. Giannakakou P, Sackett DL, Ward Y, Webster KR, Blagosklonny MV, Fojo T. p53 is associated with cellular microtubules and is transported to the nucleus by dynein. Nat Cell Biol. 2000;2(10):709-717.

9. Mabjeesh NJ, et al. 2ME2 inhibits tumor growth and angiogenesis by disrupting microtubules and dysregulating HIF. Cancer Cell. 2003;3(4):363-375.

10. Gascoigne KE, Taylor SS. Cancer cells display profound intra- and interline variation following prolonged exposure to antimitotic drugs. Cancer Cell. 2008;14(2):111-122.

11. Symmans WF, et al. Paclitaxel-induced apoptosis and mitotic arrest assessed by serial fine-needle aspiration: implications for early prediction of breast cancer response to neoadjuvant treatment. Clin Cancer Res. 2000;6(12):4610-4617.

12. Milross CG, Mason KA, Hunter NR, Chung WK, Peters LJ, Milas L. Relationship of mitotic arrest and apoptosis to antitumor effect of paclitaxel. J Natl Cancer Inst. 1996;88(18):1308-1314.

13. Orr GA, Verdier-Pinard P, McDaid H, Horwitz SB. Mechanisms of Taxol resistance related to microtubules. Oncogene. 2003;22(47):7280-7295.

14. Giannakakou P, et al. Paclitaxel-resistant human ovarian cancer cells have mutant beta-tubulins that exhibit impaired paclitaxel-driven polymerization. J Biol Chem. 1997;272(27):17118-17125.

15. Giannakakou P, et al. A common pharmacophore for epothilone and taxanes: molecular basis for drug resistance conferred by tubulin mutations in human cancer cells. Proc Natl Acad Sci U S A. 2000;97(6):2904-2909.

16. Berrieman HK, Lind MJ, Cawkwell L. Do betatubulin mutations have a role in resistance to chemotherapy? Lancet Oncol. 2004;5(3):158-164.

17. Gottesman MM, Fojo T, Bates SE. Multidrug resistance in cancer: role of ATP-dependent transporters. Nat Rev Cancer. 2002;2(1):48-58.

18. Zhu Y, et al. Inhibition of ABCB1 expression overcomes acquired docetaxel resistance in prostate cancer. Mol Cancer Ther. 2013;12(9):1829-1836.

19. Robey RW, Pluchino KM, Hall MD, Fojo AT, Bates SE, Gottesman MM. Revisiting the role of $\mathrm{ABC}$ transporters in multidrug-resistant cancer. Nat Rev Cancer. 2018;18(7):452-464.

20. Duran GE, et al. Cabazitaxel is more active than first-generation taxanes in $\mathrm{ABCB} 1(+)$ cell lines due to its reduced affinity for P-glycoprotein. Cancer Chemother Pharmacol. 2018;81(6):1095-1103.

21. Bugde $P$, et al. The therapeutic potential of targeting $\mathrm{ABC}$ transporters to combat multi-drug resistance. Expert Opin Ther Targets. 2017;21(5):511-530.

22. Galletti G, et al. ERG induces taxane resistance in castration-resistant prostate cancer. Nat Commun. 2014;5:5548.

23. Yoshino T, et al. Bcl-2 expression as a predictive marker of hormone-refractory prostate cancer treated with taxane-based chemotherapy. Clin Cancer Res. 2006;12(20 pt 1):6116-6124.

24. O'Neill AJ, et al. Characterisation and manipulation of docetaxel resistant prostate cancer cell lines. Mol Cancer. 2011;10:126.

25. Domingo-Domenech J, et al. Suppression of acquired docetaxel resistance in prostate cancer through depletion of notch- and hedgehogdependent tumor-initiating cells. Cancer Cell. 2012;22(3):373-388.

26. Vidal SJ, et al. A targetable GATA2-IGF2 axis confers aggressiveness in lethal prostate cancer. Cancer Cell. 2015;27(2):223-239.

27. Darshan MS, et al. Taxane-induced blockade to nuclear accumulation of the androgen receptor predicts clinical responses in metastatic prostate cancer. Cancer Res. 2011;71(18):6019-6029.

28. Zhu ML, Horbinski CM, Garzotto M, Qian DZ, Beer TM, Kyprianou N. Tubulin-targeting chemotherapy impairs androgen receptor activity in prostate cancer. Cancer Res. 2010;70(20):7992-8002.

29. Antonarakis ES, et al. Randomized, noncomparative, phase II trial of early switch from docetaxel to cabazitaxel or vice versa, with integrated biomarker analysis, in men with chemotherapy-naïve, metastatic, castration-resistant prostate cancer. JClin Oncol. 2017;35(28):3181-3188.
30. Thadani-Mulero M, et al. Androgen receptor splice variants determine taxane sensitivity in prostate cancer. Cancer Res. 2014;74(8):2270-2282.

31. Zhang G, et al. Androgen receptor splice variants circumvent AR blockade by microtubule-targeting agents. Oncotarget. 2015;6(27):23358-23371.

32. Vrignaud $P$, et al. Preclinical antitumor activity of cabazitaxel, a semisynthetic taxane active in taxane-resistant tumors. Clin Cancer Res. 2013;19(11):2973-2983.

33. Shalli K, Brown I, Heys SD, Schofield AC. Alterations of beta-tubulin isotypes in breast cancer cells resistant to docetaxel. FASEB J. 2005;19(10):1299-1301.

34. Hari M, et al. Paclitaxel-resistant cells have a mutation in the paclitaxel-binding region of beta-tubulin (Asp26Glu) and less stable microtubules. Mol Cancer Ther. 2006;5(2):270-278.

35. Yu Y, et al. Inhibition of spleen tyrosine kinase potentiates paclitaxel-induced cytotoxicity in ovarian cancer cells by stabilizing microtubules. Cancer Cell. 2015;28(1):82-96.

36. Zheng Y, et al. Tuning microtubule dynamics to enhance cancer therapy by modulating FERmediated CRMP2 phosphorylation. Nat Commun. 2018;9(1):476.

37. Yang H, et al. Paclitaxel sensitivity of ovarian cancer can be enhanced by knocking down pairs of kinases that regulate MAP4 phosphorylation and microtubule stability. Clin Cancer Res. 2018;24(20):5072-5084.

38. Liu C, et al. Functional p53 determines docetaxel sensitivity in prostate cancer cells. Prostate. 2013;73(4):418-427.

39. Zhong B, et al. Induction of clusterin by AKT--role in cytoprotection against docetaxel in prostate tumor cells. Mol Cancer Ther. 2010;9(6):1831-1841.

40. Thadani-Mulero M, Nanus DM, Giannakakou P. Androgen receptor on the move: boarding the microtubule expressway to the nucleus. Cancer Res. 2012;72(18):4611-4615

41. de Leeuw R, et al. Novel actions of next-generation taxanes benefit advanced stages of prostate cancer. Clin Cancer Res. 2015;21(4):795-807.

42. Nguyen HM, et al. LuCaP prostate cancer patient-derived xenografts reflect the molecular heterogeneity of advanced disease and serve as models for evaluating cancer therapeutics. Prostate. 2017;77(6):654-671. 